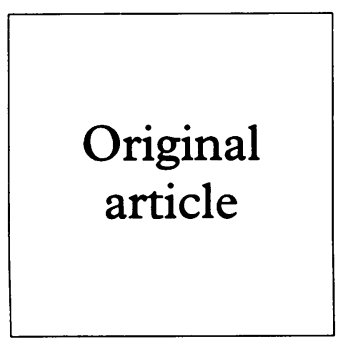

\title{
Comparative periurethral bacteriology of uncircumcised and circumcised males
}

\author{
F Serour, Z Samra, Z Kushel, A Gorenstein, M Dan
}

Background: It has been established that lack of circumcision increases the risk of urinary tract infection in infants. During the first six months, the presence of foreskin is associated with a greater quantity and a higher concentration of uropathogens in the periurethral area. Very little is known about this association in older males.

Objective: To compare the periurethral bacteriology of uncircumcised healthy males of more than one year of age.

Methods: The periurethral area of 125 uncircumcised and 46 circumcised healthy males (mean age, $26 \cdot 5$ and $28 \cdot 3$ years, respectively) was swabbed and cultured for facultative and anaerobic bacteria, genital mycoplasmas and Chlamydia trachomatis.

Results: Facultative Gram positive cocci predominated in both groups (62\% and $80 \%$, respectively). Pure culture of facultative Gram negative rods was more common in uncircumcised males $(17 \% v 4 \%$ in circumcised males, $\mathrm{p}=0.01)$. Streptococci, strict anaerobes and genital mycoplasmas were found almost exclusively in uncircumcised males of more than 15 years of age. No case of $C$ trachomatis was identified.

Conclusions: The higher prevalence of potential uropathogens in the subpreputial space is in accordance with a previous finding of increased risk of urinary tract infection in uncircumcised young men. Our results also support the role of the prepuce as a reservoir for sexually transmitted organisms.

(Genitourin Med 1997;73:288-290)

Keywords: circumcision; genital flora; bacteriology

\section{Introduction}

The medical interest in circumcision has focused mainly on the association between the prepuce and urinary tract infections in infants. ${ }^{1}$ Ginsburg and McCracken ${ }^{2}$ first noted that $95 \%$ of male infants with urinary tract infection were uncircumcised. Wiswell and Roscelli, ${ }^{3}$ in retrospective cohort studies, have documented that uncircumcised infants had a ten-fold to twenty-fold greater risk of urinary tract infection in the first few months of life. Wiswell et $a l^{4}$ have also demonstrated that during the first six months of life, the presence of a foreskin was associated with a greater number of periurethral bacteria and a greater likelihood for the presence of, as well as higher concentrations of, potentially uropathogenic organisms. More recently, Spach et $a l^{5}$ observed that lack of circumcision increased the risk of urinary tract infection in young men. However, very little is known about the subpreputial bacteriology of healthy males during childhood and adulthood. The purpose of the present study was to address the above question and to examine whether the healthy prepuce can serve as a reservoir for sexually transmitted organisms, such as genital mycoplasmas and Chlamydia trachomatis.

\section{Material and methods}

Healthy uncircumcised males, participating in a programme of ritual circumcision among new immigrants, were included in the study. A group of circumcised individuals admitted for minor surgery served as controls. None had received antimicrobial agents in the preceding month, and no external cleansing was done before sampling. In the uncircumcised males, the preputial sac was sampled, while in the circumcised ones, the periurethral area was swabbed. Three swabs were obtained from each subject and immediately transferred to the laboratory. For general bacterial cultures, a plain cotton wool swab was transported in modified Stuart's transport medium and plated onto four plates: phenylethanol agar (Difco Laboratories, Detroit, USA) for isolation of facultative Gram positive organisms; MacConkey agar (Difco Laboratories, Detroit, USA) for isolation of facultative Gram negative organisms; triptic soy agar supplemented with $5 \%$ defibrinated sheep blood (El-Dan Technologies, Jerusalem, Israel) for isolation of fastidious organisms; and Columbia agar supplemented with $5 \%$ human blood (Becton-Dickinson Microbiology Systems, Cockeysville, MD, USA) for isolation of anaerobes. Organisms were identified by standard microbiological methods. For isolation of genital mycoplasmas, a swab was placed into modified Stuart's transport medium. Cultures were performed on A3 or A7 Medium (broth and agar). For isolation of Chlamydia trachomatis, a calcium alginate swab was placed into a vial containing $0.2 \mathrm{M}$ 
sucrose in a $0.02 \mathrm{M}$ phosphate buffer with $10 \mu \mathrm{g} / \mathrm{ml}$ of gentamicin and $1 \mu \mathrm{g} / \mathrm{ml}$ of amphotericin B (Chlamydia Transwab, Medical Wire \& Equipment, Co, Corsham, W.its, UK). The samples were frozen at $-70^{\circ} \mathrm{C}$ until cultured. Specimens were inoculated on cycloheximide treated McCoy's cells. Chlamydia inclusion bodies were detected by iodine staining after 48 and 72 hours of incubation. Statistical comparisons were made using the $\chi^{2}$ test and Fisher's exact test.

\section{Results}

A total of 125 uncircumcised individuals, aged 2 to 54 years (mean 26.5) and 46 circumcised subjects (mean age 28.3) were included in the study. Eighteen per cent were 15 years' old or younger. None had complaints or findings suggestive of sexually transmitted disease or urinary tract infection.

At least one isolate was detected in $94.5 \%$ of uncircumcised and all of the circumcised males. The distribution of different bacteria that were isolated is shown in the table. Facultative Gram positive bacteria were isolated in $77.6 \%$ of uncircumcised and $95.6 \%$ of circumcised males $(p=0 \cdot 01)$. Pure facultative Gram positive organisms were documented in $48 \%$ of uncircumcised and $78 \%$ of circumcised individuals $(p=0.001)$. Facultative Gram negative rods were isolated in $24 \%$ of uncircumcised and $22 \%$ of circumcised individuals. Pure facultative Gram negative bacteria were observed in $17.6 \%$ of uncircumcised and $4.3 \%$ of circumcised males $(p=0.01)$. More than two Gram negative organisms were isolated from $5.6 \%$ of uncircumcised and $2 \%$ of circumcised participants. Strict anaerobes were detected in $21 \%$ of uncircumcised and none of circumcised individuals $(p=0.001)$. Only one of the individuals harbouring anaerobes $(3.5 \%)$ was less than 15 years of age $(p=$ $0.01)$. Facultative Gram positive bacteria predominated in both groups $(p<0.001$ versus facultative Gram negative rods), coagulase-

Prevalence of various bacteria isolated from the periurethral area of uncircumcised and circumcised males

\begin{tabular}{lll}
\hline Organism & Uncircumcised & Circumcised \\
\hline Total number of isolates & $N=125$ & $N=46$ \\
\hline Facultative Gram positive bacteria & 171 * & 55 \\
Coagulase negative staphylococci & $106(62 \%)+$ & $44(80 \%)$ \\
Streptococci † & 92 & 44 \\
Staphylococcus aureus & 11 & 0 \\
Enterococcus spp & 1 & 0 \\
Corynebacterium spp & 1 & 0 \\
Facultative Gram negative bacteria & 1 & $11(20 \%)$ \\
Klebsiella spp & $37(22 \%)$ & 1 \\
E coli & 10 & 4 \\
Acinetobacter spp & 8 & 3 \\
Enterobacter spp & 7 & 1 \\
Proteus mirabilis & 5 & 0 \\
Citrobacter spp & 4 & 1 \\
Paeruginosa & 2 & 1 \\
Anaerobes & 1 & 0 \\
Bacteroides fragitis & $28(16 \%)$ & \\
Bacteroides spp & 2 & ND \\
Mycoplasmas & 26 & \\
\hline
\end{tabular}

* Mycoplasmas excluded.

+Percentages are of total number of isolates.

†Nine non-haemolytic and two alpha haemolytic.

ND $=$ not done. negative staphylococci being the most common single species and the only Gram positive bacterium in circumcised males. Streptococci were significantly more common in the uncircumcised than in the circumcised group $(p=$ $0.04)$. All carriers of these organisms were 20 years of age or older. Most of the streptococcal isolates $(\sim 80 \%)$ were non-haemolytic. Among facultative Gram negative rods Klebsiella and Escherichia coli predominated in uncircumcised males, while $E$ coli and Acinetobacter were most common organisms in the circumcised; no difference was observed between the two groups of individuals in their isolation rate. Streptococci and anaerobes were isolated in all instances in combination with coagulase negative staphylococci. While coagulase negative staphylococci were isolated in pure culture as often as mixed cultures (49 versus 43 cases, respectively), facultative Gram negative organisms were recovered in pure culture twice as often as in mixed cultures (19 versus 10 cases, respectively). Other than the streptococci and anaerobes, all other species were similarly distributed in the two age groups (up to and above 15 years), although the proportion of Gram negative organisms was somewhat higher in the young age group (37\% versus $25 \%$ in the adult group). The four uncircumcised individuals who harboured genital mycoplasmas (Mycoplasma hominis in one, Ureaplasma urealyticum in two and both species in the fourth case) were also colonised by coagulase negative staphylococci. Their age ranged between 20 and 39 years. In no case was $C$ trachomatis isolated.

\section{Discussion}

Facultative Gram positive bacteria (mainly coagulase negative staphylococci) predominated both in circumcised and uncircumcised individuals. There was no difference between the two groups in the isolation rate of facultative Gram negative organisms. However, when pure growth was considered, facultative Gram negative rods which are potential uropathogens were significantly more common in uncircumcised males. The findings is not necessarily fortuitous and might be related to the increased prevalence of urinary tract infection in uncircumcised individuals. ${ }^{5}$ One may speculate that when Gram negative organisms are the only colonisers of the preputial space, they achieve higher concentrations; this quantitative difference may contribute to the development of urinary tract infection.

Of special interest is the observation that streptococci and anaerobes were found almost exclusively in uncircumcised males more than 15 years of age. This finding would suggest that these organisms, which are common inhabitants of the female genital tract, might have been acquired by sexual transmission. The anal origin of the organisms can be excluded, because if that were the case, their occurrence would not be age related. Indeed, in a previous study conducted among boys less than 14 years of age, anaerobes were rarely detected in both circumcised and uncircum- 
cised individuals. ${ }^{6}$ Thus, the prepuce may serve as a reservoir for sexually transmitted organisms. Indeed, the association between lack of male circumcision and susceptibility to "conventional" sexually transmitted diseases, particularly those that produce ulcerations, has been reported by several authors..$^{7-9}$ More recently, a substantial body of evidence was presented linking non-circumcision in men with risk for human immunodeficiency virus (HIV) infection. ${ }^{10}$ It was even recommended that "consideration should be given to male circumcision as an intervention to reduce HIV transmission". ${ }^{10}$

To conclude, the findings of the present study support a potential role for the prepuce in the pathogenesis of urinary tract infection in males even beyond infancy. We have also contributed to the understanding of the association between non-circumcision and sexually transmitted diseases.
1 Wiswell TE. Prepuce presence portends prevalence of potentially perilous periurethral pathogens. F Urol 1992; 148:739-42.

2 Ginsburg SM, McCracken GH. Urinary tract infections in young infants. Pediatrics 1982;69:409-12.

3 Wiswell TE, Roscelli JP. Corroborative evidence for the decreased incidence of urinary tract infections in circumcised male infants. Pediatrics 1986;78:96-9.

4 Wiswell TE, Miller GM, Gelston HM, Jones SK, Clemmings AF. Effect of circumcision status on periurethral bacterial flora during the first year of life. $f$ Pediatr 1988;113:442-6.

5 Spach DH, Stapleton AE, Stamm WE. Lack of circumcision increases the risk of urinary tract infection in young men. $\Im A M A$ 1992;267:679-81.

6 Glenon J, Ryan PJ, Kaene CT, Rees JPR. Circumcision and periurethral carriage of Proteus mirabilis in boys. Arch Dis Child 1988;63:556-7.

7 Taylor PK, Rodin P. Herpes genitalis and circumcision. $B$ f Venereal Dis 1975;51:274-7.

8 Parker SW, Stewart AJ, Wren MN, Gollow MN, Straton JA. Circumcision and sexually tranmissible disease. Me $\mathcal{F}$ Aust 1983;2:288-90.

9 Smith GL, Greenup R, Takafuji ET. Circumcision as a risk factor for urethritis in racial groups. Am $\mathcal{F}$ Public Health 1987;77:452-4.

10 Moses S, Plummer FA, Bradley JE, Ndinya-Achola JO, Nagelkerke NJD, Roland AR. The association between lack of male circumcision and risk for HIV infection: review of the epidemiological data. Sex Trans Dis 1994; 21:201-10.

Historical
vignette

\section{Podophyllin - the early days}

Records of the medicinal use of podophyllum and its derivatives go back for 200 years, long before they became known for the treatment for genital warts. Podophyllum peltatum is a flowering perennial, widely spread throughout North America. The Indians knew of the medical properties of its rhizome, and this knowledge reached the early colonists and, in due course, their doctors. Podophyllum was regarded as an excellent emetic and cathartic, at a time when purging was an important part of medical treatment, and also as a hepatic stimulant. It was included in the first United States Pharmacopoeia, published in 1820. Podophyllin resin was separated from the crude rhizome in 1835, and was thereafter preferred as a more refined product. Another species, Podophyllum emodi, which grows in the Himalayas, was described in 1839. It had similar properties to $P$ peltatum, but it was not widely used; most podophyllum preparations, even in India, were imported from the United States.

The drug soon became popular among the "eclectic" sect of practitioners, who made special use of indigenous plant remedies. It was said that podophyllin was not only a cathartic and cholagogue, but of value in respiratory, renal, rheumatic, and menstrual disorders. It was found to be irritating when applied to the skin, and this led to its use as a counterirritant for the treatment of neuralgia and synovitis, although the resulting cutaneous inflammation could be severe. There can be no doubt that in the United States at this time podophyllin was regarded as a valuable drug by both doctors and laymen. It was manufactured on a large scale: in 1860, 2000 kilograms were marketed. As information about the "new American remedy" crossed the Atlantic, review articles appeared in the English medical press in 1861 and 1862, and The Lancet published enthusiastic letters from readers who had prescribed the drug. It soon appeared in the British Pharmacopoeia in the form of a powder and tincture, and non-official preparations in which the podophyllin was mixed with other drug were developed.

In the mid 19th century, pharmacology was in a chaotic state. Many botanical and chemical substances were available, and polypharmacy, with long winded prescriptions, was the rule. The action of some individual drugs was understood, but podophyllin was not one of these. By the end of the century interest had waned, and its use had diminished to the treatment of constipation (when it was usually combined with other products) and of so called "functional disorders of the liver". The latter was a vaguely defined syndrome consisting of headache, depression, anorexia, abdominal discomfort, and constipation. Podophyllin was believed, incorrectly, to increase the flow of bile and had a reputation, particularly among the laity, for the relief of the condition; it was a constituent of several proprietary remedies, of which Carter's Little Liver Pills (a combination of podophyllum and aloes) wer the best known. Some individuals took regular smal doses of these products indefinitely.

Eventually, the indications for podophyllin were reduced to its use as a hydragogue purgative, and even here, in due course, it was replaced by less violent medicaments. Its escharotic properties had not been completely forgotten, however. For many years urologists in New Orleans had treated genital warts with podophyllin, but the treatment was not generally? known until 1942, when Isaac Kaplan, working with the United States Army in New Orleans, reported that condylomata acuminata regressed after applications of $25 \%$ podophyllin suspended in mineral oil. At this point "the door opened and let the future in".

J D ORIEL 